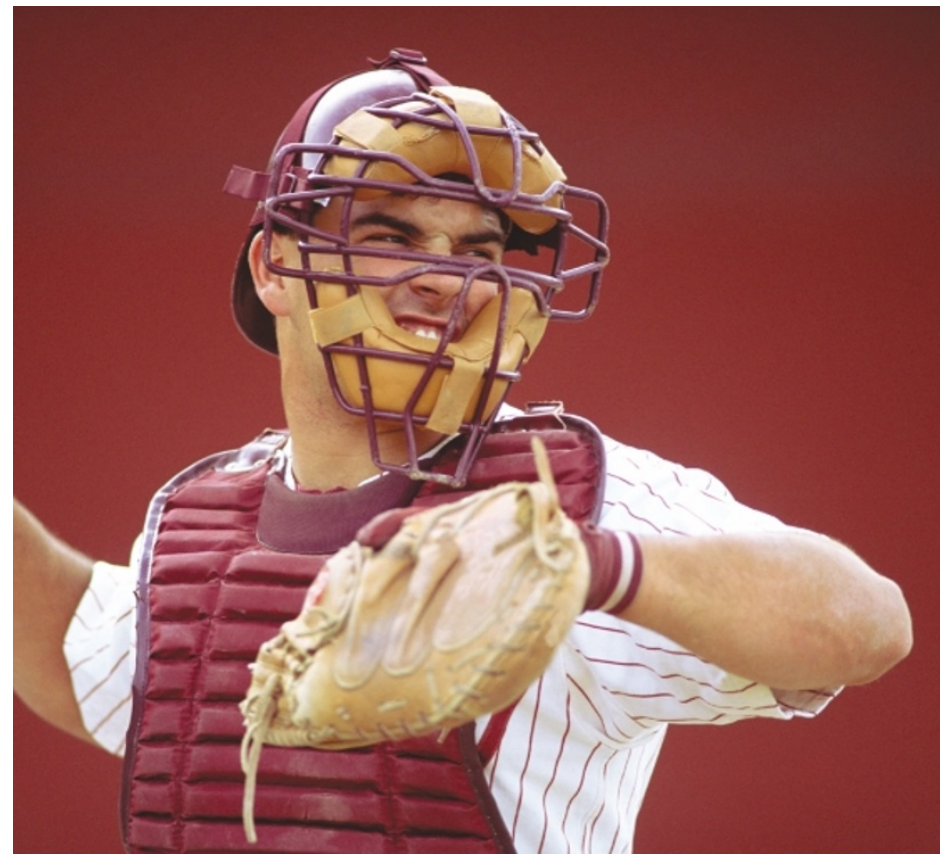

VACCINES

\title{
HIV: effective protection?
}

Mucosal surfaces are the natural sites of transmission of many viruses - for HIV, transmission occurs at the genital and gastrointestinal surfaces. The gut mucosa is also a major reservoir for $\mathrm{HIV}$, from which viruses can seed the bloodstream. Therefore, an HIV vaccine will probably need to generate effective mucosal immunity. In Nature Medicine, Belyakov and colleagues report that intra-rectal administration of a synthetic peptide vaccine induces specific cytotoxic $\mathrm{T}$ lymphocytes (CTLs) in the gut mucosa of macaques. Although the CTL response could not prevent infection after intrarectal challenge, it had a positive impact on the course of infection.

This study is the first to directly compare mucosal and systemic immunization with the same vaccine in primates, and to assess the contribution of mucosal CTLs in clearing the mucosal viral reservoir. The peptide vaccine consisted of a mixture of four peptides, each containing a cluster of overlapping HIV-1 helper epitopes, and various CTL epitopes from simian immunodeficiency virus (SIV) Gag and Pol. Macaques were immunized either intra-rectally or subcutaneously in two cycles of four vaccine immunizations. In intra-rectally immunized animals, CTL responses were detected in both mucosal lymphoid sites and systemic lymphoid sites. By contrast, subcutaneous immunization predominantly elicited CTLs in the systemic compartment.
At 8 weeks after the immunizations, macaques were given an additional dose of vaccine, and 2 weeks later, were challenged intra-rectally with a pathogenic strain of simian/ HIV, SHIV-Ku2. Animals immunized intra-rectally, in contrast to subcutaneously immunized animals, had plasma viral loads which rapidly fell below the level of detection.

So, does the mucosal CTL response correlate with control of infection at this site? At autopsy, 200 days after challenge with SHIV-Ku2, the CTL responses and viral loads in the gut mucosa were assessed. None of the intra-rectally immunized animals had detectable virus in the colon or jejenum, whereas subcutaneously immunized animals all had detectable virus.

This report provides the first evidence from primate studies in support of the idea that mucosal CTL responses are as significant as systemic responses in protection against mucosal transmission of HIV/SIV, and suggests that this will be an important consideration in the development of efficacious AIDS vaccines.

Elaine Bell

\section{(1) References and links} ORIGINAL RESEARCH PAPER Belyakov, I. M. et $a$ al. Mucosal AIDS vaccine reduces disease and viral load in gut reservoir and blood after mucosal infection of macaques. Nature Med. 7 1320-1326 (2001)

\section{WEB SITE}

Jay Berzofsky's lab: http://www-dcs.nci.nih.gov/ resdir/person_index.cfm?p_id=64

\section{IN BRIEF}

\section{IMMUNE REGULATION}

NKG2D receptors induced by IL-15 costimulate CD28negative effector CTL in the tissue microenvironment.

Roberts, A. I. et al. J. Immunol. 167, 5527-5530 (2001)

The activation and regulation of naive $\mathrm{T}$ cells is well understood, but how are effector $\mathrm{T}$ cells regulated when they reach peripheral tissues? This study suggests that CTL responses in tissues can be fine-tuned by stress or inflammation. Roberts and colleagues report that NKG2D, the natural killer receptor for stress-induced MHC class I chain-like gene A and B molecules, is a potent co-stimulatory molecule for freshly isolated intestinal CTLs. Moreover interleukin-15, which is released by inflamed intestinal epithelium, selectively upregulates NKG2D expression and function.

\section{IMMUNE REGULATION}

$B$ cells and professional APCs recruit regulatory T cells via CCL4.

Bystry, R. S. et al. Nature Immunol. 12, 1126-1132 (2001)

In this study, the chemokine and chemokine receptor geneexpression profile of activated B cells was assessed. B cells activated through the BCR, but not by lipopolysaccharide, were shown to strongly upregulate CCL4 expression. In vitro, this chemokine was shown to preferentially attract $\mathrm{CD} 4^{+} \mathrm{CD} 25^{+}$regulatory $\mathrm{T}$ cells, rather than conventional helper T cells. Also, in vivo depletion of CCL4 led to enhanced B-cell responses and autoantibody production.

\section{INNATE IMMUNITY}

Drosophila Toll is activated by Gram-positive bacteria through a circulating peptidoglycan recognition protein. Michel, T. et al. Nature 414, 756-759 (2001)

The Toll signalling pathway in Drosophila is activated in response to infection by Gram-positive bacteria and fungi, but the Toll receptor does not seem to recognize pathogens directly. Here, a new mutation, seml, was idenitifed that acts upstream of Toll to block responses to Gram-positive bacteria but not fungi. seml was mapped to a gene that encodes a secreted peptidoglycanrecognition protein.

\section{PARASITE IMMUNOLOGY}

Apoptotic mimicry by an obligate intracellular parasite downregulates macrophage microbicidal activity.

de Freitas Balanco, J. M. et al. Curr. Biol. 11, 1870-1873 (2001)

Phagocytosis of microbes by macrophages normally triggers microbicidal activity, but this does not happen in the case of Leishmania parasites - they survive and multiply inside macrophages. This study indicates that Leishmania amastigotes might avoid activating macrophages by mimicing apoptotic cells. Amastigotes express phosphatidylserine on their suface, which leads to phagocytosis by macrophages, production of the anti-inflammatory cytokines TGF- $\beta$ and IL-10, and suppression of $\mathrm{NO} \cdot$ production. 